\title{
What better measure than ribosome synthesis?
}

\author{
Dipayan Rudra and Jonathan R. Warner ${ }^{1}$ \\ Department of Cell Biology, Albert Einstein College of Medicine, Bronx, New York 10461, USA
}

Cells grow; cells divide. Some cells grow without dividing: neurons, or oocytes. Some cells divide without growing: developing zygotes. For most cells, however, growth and division are coupled, thereby maintaining cell size within narrow limits. The basis of this coupling has been elusive.

Cell growth was the subject of some of the earliest molecular biological investigations when it was shown that, on a variety of media, the ribosome content of Escherichia coli was proportional to the growth rate (Maaloe and Kjeldgaard 1966). Soon thereafter, the study of growth was overshadowed by the study of gene expression with its emphasis on the regulated transcription of individual genes.

More recently, the recognition that growth and cell division are so closely intertwined has led to new interest in the study of growth per se, as suggested by the recent publication of two reviews (Kozma and Thomas 2002; Saucedo and Edgar 2002) and a monograph, Cell Growth: Control of Cell Size (Hall et al. 2004). In considering cell growth and division, two points are paramount: (1) Cell growth requires the synthesis of proteins; the synthesis of proteins requires ribosomes. Thus, ultimately the control of cell growth must involve the control of ribosome synthesis. (2) A cell must somehow match the replication of its genome, with the implicit decision to divide, to its size, or perhaps to its predicted size at the time division will occur.

These two aspects have often been considered in parallel, the studies of macromolecular synthesis, transcription, and translation proceeding with little care for their implications for cell division, and the studies of the intricate dance of cyclins that powers the cell cycle relatively unconcerned with the macromolecular events within the cell that ultimately drive the decision to divide. Those involved in the cell cycle, however, have been more attentive, if no more successful at understanding the mechanisms controlling the relationship between growth and division. Thus, the dean of the cell cycle field recently wrote, "The existence of a 'size con-

${ }^{1}$ Corresponding author.

E-MAIL warner@aecom.yu.edu; FAX (718) 430-8574.

Article and publication are at http://www.genesdev.org/cgi/doi/10.1101/ $\operatorname{gad} .1256704$ trol' is well known ... but it has been remarkably resistant to molecular analysis. ... It is a vital link coordinating cell growth with periodic events of the cycle." (Mitchison 2003).

\section{Genetic approaches to cell size control}

It has long been known for Saccharomyces cerevisiae that cell size is proportional to ploidy, and also depends on nutritional state. Cells growing slowly in poor medium are small; shifting cells from rich to poor medium decreases the critical size, and vice versa, an effect that is mediated in part by the Ras/PKA pathway (for review, see Jorgensen et al. 2004b). But what are the signals that designate size?

An early insight into this problem derived from the analysis by Hartwell and coworkers (Johnston et al. 1977) of the growth of S. cerevisiae cells blocked in the division cycle, and, conversely, the cycling of cells inhibited in growth. They concluded that growth did not depend on a functioning cycle, but that some event in G1 "...cannot be completed until a critical size is attained." In short, "growth, rather than progress through the DNA-division cycle, is normally limiting for cell proliferation." The identification of wee mutants in Schizosaccharomyces pombe (Thuriaux et al. 1978) and whi mutants in S. cerevisiae (Carter and Sudbery 1980; Nash et al. 1988), with significantly smaller cells than wild type, provided a genetic approach to the problem. In $S$. cerevisiae, in which the variable part of the cell cycle is G1, such mutants were considered to be making a premature decision to replicate their DNA, and thus to "Start": to enter the next cell cycle. It is noteworthy that many such mutants had the same doubling time as wildtype cells, demonstrating that cell size is not necessarily connected to cell growth rate.

Basically similar relationships have been reported for Drosophila. Genetic manipulation to increase or decrease the rate of cell cycling alters the cell number but not the size of the imaginal disc involved (Neufeld and Edgar 1998). Similarly, deletion of the S6 kinase, a potential regulator of ribosome synthesis, leads to slowly developing, small flies with small but not fewer cells. (Montagne et al. 1999).

Yet the molecular basis of size control has remained 
obscure. A substantial contribution to this problem is reported in this issue of Genes \& Development, where Tyers and coworkers have followed up their earlier genome-wide screen for mutants affecting cell size (Jorgensen et al. 2002) with experiments that lead them to conclude that the coupling between cell growth and cell division is mediated by two genes involved in the regulation of the rate of ribosome biosynthesis (Jorgensen et al. 2004a). In short, ". . .nutrient control of the critical cell size threshold ... is communicated to the Start machinery via proximal events in ribosome biosynthesis, rather than by downstream changes in protein synthetic rate."

\section{Cell size}

Briefly, the key player in cell size homeostasis in S. cerevisiae appears to be a G1 cyclin, Cln3 (Cross 1988), that functions to coordinate Start with cell growth and with the supply of nutrients. A critical concentration of Cln3 in the nucleus results in the activation of the Cln3Cdc28 kinase. This kinase phosphorylates and releases Whi5, a negative regulator and interacting partner of the $\mathrm{SBF} / \mathrm{MBF}$ transcription factor complexes (Costanzo et al. 2004; de Bruin et al. 2004) The dissociation of Whi5 activates the SBF/MBF transcriptional machinery and leads to the activation of a set of some $\sim 120$ genes including two other G1 cyclins, CLN1 and CLN2, and the B-type cyclins CLB5 and CLB6. The Clb5 and Clb6 proteins thus formed are complexed with the negative regulator Sic1. The phosphorylation and subsequent degradation of Sic1 by the $\mathrm{Cln} 1$ and $\mathrm{Cln} 2-\mathrm{Cdc} 28$ complexes, respectively, activates Clb5/6-Cdc28 complexes to trigger "Start" and thus initiate DNA replication (for review, see Rupes 2002). Overexpression of Cln3 leads to an accelerated Start and a decrease in cell size, but with little effect on the doubling time. Conversely, deletion of CLN3 leads to a delayed start, and thus a considerable increase in cell size.

How then does the cell make the decision that sufficient $C \ln 3$ has accumulated to initiate S? There have been invocations of cytoplasmic volume, ratio of cytoplasmic to nuclear volume, rate of translation, activity of translation initiation factors, and many others (for re- view, see Rupes 2002). An intriguing observation in $S$. cerevisiae is that the $5^{\prime}$-leader of the CLN3 transcript has a small open reading frame (ORF), analogous to that of GCN4, suggesting that regulation of translation is at work. Indeed, disruption of the upstream ORF, presumably permitting far more efficient translation of $\mathrm{Cln} 3$, also leads to small cells (Polymenis and Schmidt 1997).

\section{Ribosome biosynthesis}

If cell size depends on some aspect of ribosome synthesis, it is important to consider what features distinguish the control of ribosome synthesis from most other control pathways.

(1) Ribosome synthesis requires the coordinated activities of all three RNA polymerases: Pol I for rRNA, Pol II for the ribosomal protein (RP) genes, and Pol III for $5 \mathrm{~S}$ RNA.

(2) Quantity matters. The cell needs precisely equimolar amounts of rRNA and each of the 79 RPs. Excess rRNA will not be properly assembled into a ribosome with insufficient RPs. Insufficiency of any one of the 79 RPs will, in most cases, lead to aberrant processing and insufficient ribosomal subunits.

(3) The processing of rRNA and the assembly of ribosomes requires an army of nearly 200 proteins (Fatica and Tollervey 2002), whose synthesis is regulated in tandem with, but not identically to, that of the RPs (the ribi regulon) (Gasch et al. 2000; Jorgensen et al. 2004a).

(4) Ribosome synthesis is a massive consumer within the economy of the yeast cell, where rRNA transcription represents $\sim 60 \%$ of total transcription and RP mRNA transcription represents $\sim 50 \%$ of the total Pol II transcription initiation events (Warner 1999). Thus, up- or down-regulation of ribosome synthesis can have a substantial ripple effect on the expression of other genes.

(5) Perhaps reflecting its central role in the regulation of growth, ribosome synthesis requires positive inputs from several sources, and can be repressed by negative inputs from several sources (Fig. 1).

\section{New factors in the control of cell size}

In their previous screen of the yeast gene deletion set for mutants affecting size, Jorgensen et al. (2002) identified

Figure 1. Regulation of ribosome biosynthesis in $S$. cerevisiae. Ribosome biosynthesis requires several elements working in tandem: an active TOR pathway; an active PKA pathway; sufficient amino acids; and the factors Rap1, Fh11, Ifh1, Sfp1, Sch9, Rrn3 (for rRNA), and perhaps others (see text for details). Repression of ribosome synthesis can come through inactivation of the TOR pathway, reduction of PKA activity, signaling via $\mathrm{PKC}$, deprivation of an amino acid, stress of many kinds including heat shock, free radicals, radiation, and so on (for review, see Jorgensen et al. 2004b).

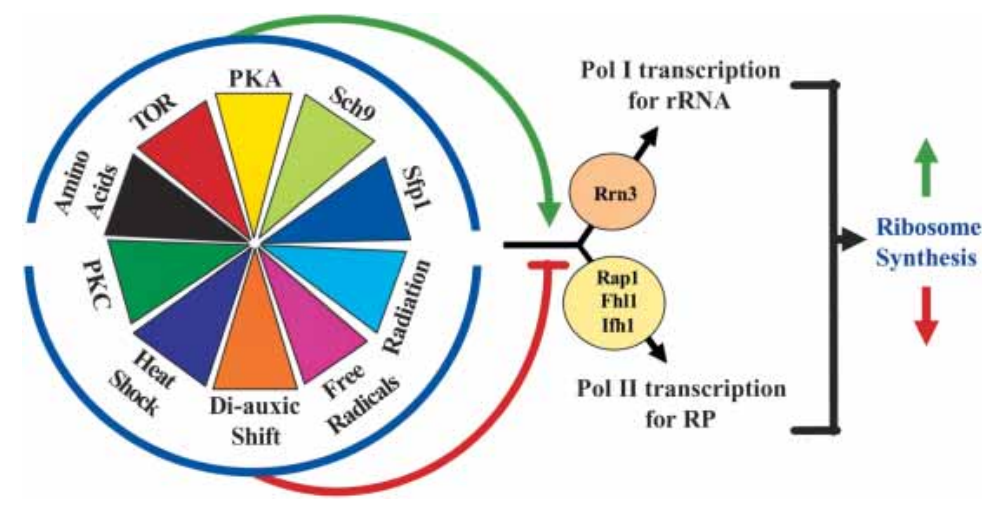


several small (whi) cell phenotypes with deletions in potential repressors of Start. The strongest whi mutants resulted from the deletion of either of two nonessential genes, SFP1 or SCH9. Sfp1 is a zinc-finger protein that has been implicated in ribosome biogenesis (Fingerman et al. 2003). Deletion of SFP1 leads not only to exceedingly small cells, but also to slow growth and to reduced levels of mRNA both for the RP genes and for the ribi regulon (Jorgensen et al. 2002). Expression of Sfp1 from the Gal promoter in such cells resulted in the prompt induction of many genes, initially those of the ribi regulon, and somewhat later the RP genes. Thus Sfp1 is implicated in the regulation of ribosome synthesis at two levels, because the ribi regulon is mediated by conserved promoter elements called RRPE and PAC (Wade et al. 2001), whereas the RP regulon is regulated by Raplpbinding sites (and others, see below).

SCH9 encodes a putative protein kinase, whose C-terminal kinase domain is $49 \%$ identical to that of human protein kinase B (PKB). Intriguingly, in both Drosophila and mouse, $\mathrm{PKB}$ is involved in cell size control by regulating the rate at which ribosomal components are produced (for reviews, see Kozma and Thomas 2002; Saucedo and Edgar 2002). In S. cerevisiae, however, Sch9 appears to be related to the ras/PKA pathway (Toda et al. 1988), which has been shown to be a key regulator of ribosome biosynthesis (Neuman-Silberberg et al. 1995). Should Sch9 be considered a PKA or a PKB? Thus, Sfp1 and Sch9 are strong candidates for the elusive proteins that couple ribosome synthesis with cell size. Indeed, overexpression of either Sfp1 or Sch9 leads to large cells. In contrast, in the absence of Sfp1 and Sch9, SBF- and MBF-dependent transcription, as well as G1-to-S transition, are accelerated after synchronous release of G1phase daughter cells obtained by centrifugal elutriation. These results further confirm the negative regulatory effect of Sfp1 and Sch9 on Start, and suggest that they act upstream to the G1/S transcriptional network. By the criterion of cell size, deletion of SFP1 is not fully epistatic to mutants that basically inactivate the $\mathrm{Cln} 3 \mathrm{regu}-$ latory elements of cell cycle control (Jorgensen et al. 2004a). A reasonable conclusion is that Sfp1 or Sch9 influences cell size by sensing nutrient availability and matching that with the rate of ribosome synthesis. It remains to be seen whether ribosome synthesis itself is a negative regulator of Start, or whether these two proteins have dual functions.

These proteins have an interesting geographical response to stress, either from inactivation of the TOR pathway or from nutritional signals through the PKA pathway. Both Sch9, usually found largely at the periphery of the vacuole, and Sfp1, usually confined to the nucleus, disperse throughout the cell. An independent genomic screen has also identified Sfp1 as being unusually responsive to stress in this way, and has provided evidence suggesting that Sfp1 is a direct regulator of RP gene expression (Marion et al. 2004). Indeed, they find that Sfp1 is present at RP genes, by ChIP analysis, but only under conditions in which transcription is occurring, although others report that enrichment of Sfp1 at
RP genes or ribi genes is barely detectable (Lee et al. 2002; Fingerman et al. 2003; Jorgensen et al. 2004a). Furthermore, the absence of Sfp1 clearly reduces the residence at RP genes of two transcription factors, Fhll and Ifh1 (Jorgensen et al. 2004a; Marion et al. 2004), that are implicated in RP gene transcription (Hermann-Le et al. 1994; Cherel and Thuriaux 1995; S. Schawalder and D. Shore, pers. comm.; D. Rudra, Y. Zhao, and J.R. Warner, in prep.). It remains to be seen how four proteins-Rap1, Fhl1, Ifh1, and Sfp1-all interact at the same site on each of the RP genes! A most interesting observation is that whenever RP transcription is repressed, and Sfp1 is dispersed from the nucleus, both Fhl1 and Ifh1 appear to concentrate in the nucleolus (Jorgensen et al. 2004a). This could be an effective way for the cell to maintain these scarce proteins readily available for use once RP transcription resumes.

Sfp1 and Sch9 appear to operate through different pathways. There is lack of genetic epistasis. Deletion of both, or simultaneously reducing their activity in a dose-dependent manner, leads to synthetic lethality. Unlike $\triangle S F P 1, \triangle S C H 9$ does not affect the nuclear localization of Fhl1 or Ifh1. Yet, ultimately these pathways must converge on ribosome biosynthesis and on cell size determination; it seems unlikely that they have no influence on each other.

It is quite remarkable that two groups have identified Sfplp as a key regulator using quite different criteria, small cell size (Jorgensen et al. 2002) or movement from nucleus to cytoplasm in response to stress (Marion et al. 2004). What remains to be seen is the role actually played by Sfp1p. Deletion of SFP1 leads to small cells that have fewer ribosomes and make protein at a slower rate (Fingerman et al. 2003). But that is true of many conditions of slow growth. Another issue is the directness of the effect. Recovery from depletion of Sfp1 initiates with increased transcription of the ribi regulon, the genes responsible for ribosome processing/assembly, with the RP genes lagging significantly behind (Jorgensen et al. 2004a). Results of over- or underexpression of Sfplp on transcription of reporter constructs carrying the signature RRPE and PAC elements of the ribi regulon confirm a relatively direct effect (Fingerman et al. 2003). Does that mean that the Sfp1 effect on transcription of the ribi regulon is primary, with the effects on RP transcription being a secondary response to that? A very recent report finds that cells deprived of phosphorous or uracil specifically down-regulate the RP but not the ribi regulon (Saldanha et al. 2004). Where will we find Sfp1 under these conditions?

The notion of Sfp1, and to a lesser extent Sch9, linking cell size determination with the regulation of ribosome biosynthesis is intriguing, as far as it goes. Indeed, for a cell undertaking the perilous steps of replicating its genome and subsequently dividing into two daughter cells, what better measure of its own health than vigorous and efficient ribosome synthesis? Yet, in the case of Sfp1, we see the opposite story: poor and inefficient ribosome synthesis is coupled to premature passage through Start. In contrast, other mutations that slow ribosome biosynthe- 
sis and slow growth, for example, deletions of a RP gene or of the RP gene transcription factor Fhllp, have little effect on cell size (Jorgensen et al. 2004a).

This suggests that Sfp1 is part of a more subtle strategy: under positive growth conditions, the cell has an interest in vigorous ribosome biosynthesis to enable rapid growth and at the same time a delay in cell division to permit the cell to grow to an optimal size. One can postulate that (in response to signals from Sch9?) Sfp1 does both, through interacting with the RP (and rRNA?) genes and through delaying Start, by means we do not yet know. Then when conditions deteriorate, in response to stress, or as cells approach late log phase, Sfp1 retreats from the nucleus, and ribosome synthesis ceases in order to provide more resources for the cell to respond to suboptimal conditions and to approach stationary phase (Ju and Warner 1994; Gray et al. 2004).

\section{Interesting results raise interesting questions}

Sfpl is a putative transcription factor. How does it activate both the ribi and the RP regulons, which have different regulatory sequences? Does Sfpl itself have transcriptional activity, or does it facilitate the activity of other factors, such as Fhll and Ifh1? How is the relocalization of Sfp1 brought about? Is it the cause or the consequence of the stress-related repression of RP gene transcription?

Sch9 is a putative kinase. What are its substrates? Is Sfp1 a substrate? What does the activity of Sch9 depend on? How is it regulated by stress? What is the implication of Sch9 being localized at the vacuolar surface? Although overexpression of Sch9 partially suppresses mutations in the ras/PKA pathway (Toda et al. 1988), their pathways seem to be parallel rather than redundant (Denis and Audino 1991). What is their relationship? The deletion of SCH9 leads to extended longevity of cells in minimal medium (Fabrizio et al. 2001). Is this related to their reduced investment in ribosome biosynthesis?

The arguments developed above are derived from data about the levels of mRNA for the ribi regulon and the RP regulon. However, one must also consider the role of the regulation of rRNA transcription by RNA polymerase I (for review, see Grummt 2003; Moss 2004). Indeed, we do not know if the regulation of rRNA and RP synthesis occurs in parallel or if one is the primary target and, in turn, regulates the other. It is clear, however, that in almost all physiological conditions, the two are closely coupled. Regulation of transcription of Pol I in S. cerevisiae is largely through the transcription factor Rrn3p (Peyroche et al. 2000; Claypool et al. 2004). Do Sfp1 and Sch9 play a role in the regulation of Rrn3 and/or rRNA transcription? What role does rRNA transcription play in cell size determination?

A number of proteins involved in ribosome synthesis and assembly appear to be associated with the replication apparatus. The ORC complex interacts with several, including Nog1, Nop5, and Nop7 (Du and Stillman 2002). Noc3, which is required for rRNA processing, serves as a key connection between the ORC and other replication initiation proteins (Zhang et al. 2002). Does the vigorous ribosome synthesis of rapidly growing cells starve the replication apparatus of these essential proteins? On the other hand, one might expect that if the absence of Sfp1 leads to a very low level of synthesis of these ribi proteins, replication would be delayed, leading to larger cells. Paradoxes abound.

\section{Growth control and ribosome synthesis in metazoa}

Do these observations coupling ribosome biosynthesis to cell size and the cell cycle have implications for higher organisms? Indeed, there has been an increasing appreciation of the role of ribosome biosynthesis in cell growth and its regulation, cell cycle control, and apoptosis in higher organisms. Perhaps the most telling experiment was carried out by Thomas and coworkers (Volarevic et al. 2000). They generated a mouse in which selective induction of cre led to the deletion of both genes for ribosomal protein S6 specifically in the liver. In spite of the lack of $40 S$ ribosome synthesis in their livers, 605 ribosome synthesis continued and the mice survived for several weeks. Their livers could respond to fasting and refeeding cycles, in which the liver changes in mass nearly twofold. However, whereas partial hepatectomy in normal animals leads to rapid regrowth and cell division, the livers of mice defective in the production of $40 \mathrm{~S}$ ribosomal subunits did not regrow and showed no signs of cell division. Thus, in this case functional ribosome synthesis appears to be essential for initiation of the cell division cycle.

\section{Ribosome synthesis and cancer/apoptosis}

It has been known for some time that key regulatory molecules of the cell influenced and were influenced by ribosome synthesis. Thus, the interaction of $\mathrm{Rb}$ with $\mathrm{UBF}$, a key factor in the transcription of rRNA, was shown to down-regulate the transcription by RNA polymerase I (Cavanaugh et al. 1995). MDM2, a key regulator of p53 turnover in mouse cells, is bound by ribosomal protein L5 (Marechal et al. 1994).

Yet only recently have specific observations linked ribosome synthesis or ribosomal components to apoptosis or to cancer. Bop1 is a mouse protein that is essential for rRNA processing. A dominant-negative allele of Boplp leads to a strong cell cycle arrest at the G1/S transition, dependent on p53 (Pestov et al. 2001). Ribosomal protein L11 has an affinity for the HDM2 protein (the human version of MDM2), whose usual function is to facilitate the degradation of $\mathrm{p} 53$. When overexpressed, or when it has no rRNA to bind to, L11 binds HDM2, leading to accumulation of $\mathrm{p} 53$, and the cells enter the apoptotic pathway (Lohrum et al. 2003; Jin et al. 2004). In this way, the cells can respond to aberrant nucleolar activity by undergoing apoptosis. Indeed, a recent analysis of damage to the nucleolus has led to the suggestion that "a p53 response, rather than being induced, has to be constantly prevented by a fully functioning nucleolus" (Rubbi and 
Milner 2003). Strikingly, the nucleolar protein p19 Arf, another partner that binds MDM2 to initiate apoptosis, interacts also with the nucleolar ribosome assembly factor B23 and seems to inhibit rRNA processing (Sugimoto et al. 2003; Bertwistle et al. 2004).

Surprisingly, in the zebrafish haploinsufficiency for any of several ribosomal proteins led to unusual malignant tumors of the peripheral nerve sheath (Amsterdam et al. 2004). This is a distinctly different situation from that of Drosophila, in which haploinsufficiency of RP genes generally leads to "minutes," relatively normal flies with minute bristles (Lambertsson 1998), although haploinsufficiency for S6 can lead to hematopoietic overgrowth (Watson et al. 1992; Stewart and Denell 1993).

Aside from the work on S6 described above, there is a surprising dearth of reports either of natural conditions of RP haploinsufficiency in the human population, or of the effects of the deletion of RP genes in mice. The single classical case is that of Diamond-Blackfan anemia, in which a variety of mutations in the gene for ribosomal protein S19 have been identified in $25 \%$ of the cases (Draptchinskaia et al. 1999). These mutations lead to an anemia with variable penetrance and to an increased incidence of leukemia. A recent report characterizes the spontaneous, semidominant, homozygous lethal Bst mutation in mouse as a deletion within the gene encoding ribosomal protein L24. The resulting phenotype includes a white ventral midline spot, a kinked tail, and other skeletal abnormalities, hardly what one might predict from the mutation of such a critical gene /Oliver et al. 2004). Although there is a clear inhibition of rRNA processing in mutant cells, there is little evidence of apoptosis, as one might expect based on the L11 results discussed above. It should be noted, however, that in yeast, L24 is one of the few RPs that is not essential for growth (Baronas Lowell and Warner 1990).

Is the lack of RP haploinsufficiency in mammals a case of "the dog that didn't bark"? Perhaps the haploinsufficiency of an RP leads to such serious defects that a mammalian organism is inviable, either because of fundamental defects in development, or because the cells are programmed to apoptose if ribosome biosynthesis becomes too aberrant.

\section{Acknowledgments}

We are grateful to Elaine Elion and Gerry Johnston for a critical reading of the manuscript and to David Shore and Stephan Schawalder for communicating results in advance of publication. Work in the authors' laboratory was supported in part by grants from the Human Frontiers Program and from the NIH: GM-25532 to J.R.W. and CAI-3330 to the Albert Einstein Cancer Center.

\section{References}

Amsterdam, A., Sadler, K.C., Lai, K., Farrington, S., Bronson, R.T., Lees, J.A., and Hopkins, N. 2004. Many ribosomal protein genes are cancer genes in zebrafish. PLOS Biol. 2: 690698.
Baronas Lowell, D.M. and Warner, J.R. 1990. Ribosomal protein L30 is dispensable in the yeast Saccharomyces cerevisiae. Mol. Cell. Biol. 10: 5235-5243.

Bertwistle, D., Sugimoto, M., and Sherr, C.J. 2004. Physical and functional interactions of the Arf tumor suppressor protein with nucleophosmin/B23. Mol. Cell. Biol. 24: 985-996.

Carter, B.L. and Sudbery, P.E. 1980. Small-sized mutants of Saccharomyces cerevisiae. Genetics 96: 561-566.

Cavanaugh, A.H., Hempel, W.M., Taylor, L.J., Rogalsky, V., Todorov, G., and Rothblum, L.I. 1995. Activity of RNA polymerase I transcription factor UBF blocked by $\mathrm{Rb}$ gene product. Nature 374: 177-180.

Cherel, I. and Thuriaux, P. 1995. The IFH1 gene product interacts with a fork head protein in Saccharomyces cerevisiae. Yeast 11: 261-270.

Claypool, J.A., French, S.L., Johzuka, K., Eliason, K., Vu, L., Dodd, J.A., Beyer, A.L., and Nomura, M. 2004. Tor pathway regulates Rrn3p-dependent recruitment of yeast RNA polymerase I to the promoter but does not participate in alteration of the number of active genes. Mol. Biol. Cell 15: 946956.

Costanzo, M., Nishikawa, J.L., Tang, X., Millman, J.S., Schub, O., Breitkreuz, K., Dewar, D., Rupes, I., Andrews, B., and Tyers, M. 2004. CDK activity antagonizes Whi5, an inhibitor of G1/S transcription in yeast. Cell 117: 899-913.

Cross, F.R. 1988. DAF1, a mutant gene affecting size control, pheromone arrest, and cell cycle kinetics of Saccharomyces cerevisiae. Mol. Cell. Biol. 8: 4675-4684.

de Bruin, R.A., McDonald, W.H., Kalashnikova, T.I., Yates III, J., and Wittenberg, C. 2004. Cln3 activates G1-specific transcription via phosphorylation of the SBF bound repressor Whi5. Cell 117: 887-898.

Denis, C.L. and Audino, D.C. 1991. The CCR1 (SNF1) and $\mathrm{SCH} 9$ protein kinases act independently of cAMP-dependent protein kinase and the transcriptional activator ADR1 in controlling yeast ADH2 expression. Mol. Gen. Genet. 229: 395-399.

Draptchinskaia, N., Gustavsson, P., Andersson, B., Pettersson, M., Willig, T.N., Dianzani, I., Ball, S., Tchernia, G., Klar, J., Matsson, H., et al. 1999. The gene encoding ribosomal protein $\mathrm{S} 19$ is mutated in Diamond-Blackfan anaemia. Nat. Genet. 21: 169-175.

Du, Y.C. and Stillman, B. 2002. Yph1p, an ORC-interacting protein: Potential links between cell proliferation control, DNA replication, and ribosome biogenesis. Cell 109: 835-848.

Fabrizio, P., Pozza, F., Pletcher, S.D., Gendron, C.M., and Longo, V.D. 2001. Regulation of longevity and stress resistance by Sch9 in yeast. Science 292: 288-290.

Fatica, A. and Tollervey, D. 2002. Making ribosomes. Curr. Opin. Cell Biol. 14: 313-318.

Fingerman, I., Nagaraj, V., Norris, D., and Vershon, A.K. 2003. Sfp1 plays a key role in yeast ribosome biogenesis. Eukaryot. Cell 2: 1061-1068.

Gasch, A.P., Spellman, P.T., Kao, C.M., Carmel-Harel, O., Eisen, M.B., Storz, G., Botstein, D., and Brown, P.O. 2000 Genomic expression programs in the response of yeast cells to environmental changes. Mol. Biol. Cell 11: 4241-4257.

Gray, J.V., Petsko, G.A., Johnston, G.C., Ringe, D., Singer, R.A., and Werner-Washburne, M. 2004. "Sleeping beauty": Quiescence in Saccharomyces cerevisiae. Microbiol. Mol. Biol. Rev. 68: 187-206.

Grummt, I. 2003. Life on a planet of its own: Regulation of RNA polymerase I transcription in the nucleolus. Genes \& Dev. 17: 1691-1702.

Hall, M.N., Raff, M., and Thomas, G., eds. 2004. Cell growth: Control of cell size. Cold Spring Harbor Laboratory Press, 
Cold Spring Harbor, NY

Hermann-Le, D.S., Werner, M., Sentenac, A., and Thuriaux, P. 1994. Suppression of yeast RNA polymerase III mutations by FHL1, a gene coding for a fork head protein involved in rRNA processing. Mol. Cell. Biol. 14: 2905-2913.

Jin, A., Itahana, K., O'Keefe, K., and Zhang, Y. 2004. Inhibition of HDM2 and activation of p53 by ribosomal protein L23. Mol. Cell. Biol. 24: 7669-7680.

Johnston, G.C., Pringle, J.R., and Hartwell, L.H. 1977. Coordination of growth with cell division in the yeast Saccharomyces cerevisiae. Exp. Cell Res. 105: 79-98.

Jorgensen, P., Nishikawa, J.L., Breitkreutz, B.J., and Tyers, M. 2002. Systematic identification of pathways that couple cell growth and division in yeast. Science 297: 395-400.

Jorgensen, P., Rupes, I., Sharom, J.R., Schepner, L., Broach, J.R., and Tyers, M. 2004a. A dynamic transcriptional network communicates growth potential to ribosome synthesis and critical cell size. Genes \& Dev. (this issue).

Jorgensen, P., Tyers, M., and Warner, J.R. 2004b. Forging the factory: Ribosome synthesis and growth control in budding yeast. In Cell growth: Control of cell size (eds. M.N. Hall et al.), pp. 329-370. Cold Spring Harbor Laboratory Press, Cold Spring Harbor, NY.

$\mathrm{Ju}$, Q. and Warner, J.R. 1994. Ribosome synthesis during the growth cycle of Saccharomyces cerevisiae. Yeast 10: 151157.

Kozma, S.C. and Thomas, G. 2002. Regulation of cell size in growth, development and human disease: PI3K, PKB and S6K BioEssays 24: 65-71.

Lambertsson, A. 1998. The minute genes in Drosophila and their molecular functions. Adv. Genet. 38: 69-134.

Lee, T.I., Rinaldi, N.J., Robert, F., Odom, D.T., Bar-Joseph, Z., Gerber, G.K., Hannett, N.M., Harbison, C.T., Thompson, C.M., Simon, I., et al. 2002. Transcriptional regulatory networks in Saccharomyces cerevisiae. Science 298: 799-804.

Lohrum, M.A., Ludwig, R.L., Kubbutat, M.H., Hanlon, M., and Vousden, K.H. 2003. Regulation of HDM2 activity by the ribosomal protein L11. Cancer Cell 3: 577-587.

Maaloe, O. and Kjeldgaard, N.O. 1966. Control of macromolecular synthesis. W.A. Benjamin, Inc., New York.

Marechal, V., Elenbaas, B., Piette, J., Nicolas, J.C., and Levine, A.J. 1994. The ribosomal L5 protein is associated with mdm-2 and mdm-2-p53 complexes. Mol. Cell. Biol. 14: 7414-7420.

Marion, R.M., Regev, A., Segal, E., Barash, Y., Koller, D., Friedman, N., and O'Shea, E.K. 2004. Sfp1 is a stress- and nutrient-sensitive regulator of ribosomal protein gene expression. Proc. Nat. Acad. Sci. (in press).

Mitchison, J.M. 2003. Growth during the cell cycle. Int. Rev. Cytol. 226: 165-258.

Montagne, J., Stewart, M.J., Stocker, H., Hafen, E., Kozma, S.C., and Thomas, G. 1999. Drosophila S6 kinase: A regulator of cell size. Science 285: 2126-2129.

Moss, T. 2004. At the crossroads of growth control; making ribosomal RNA. Curr. Opin. Genet. Dev. 14: 210-217.

Nash, R., Tokiwa, G., Anand, S., Erickson, K., and Futcher, A.B. 1988. The WHI1+ gene of Saccharomyces cerevisiae tethers cell division to cell size and is a cyclin homolog. EMBO $T$. 7: 4335-4346.

Neufeld, T.P. and Edgar, B.A. 1998. Connections between growth and the cell cycle. Curr. Opin. Cell Biol. 10: 784790.

Neuman-Silberberg, F.S., Bhattacharya, S., and Broach, J.R. 1995. Nutrient availability and the RAS/cyclic AMP pathway both induce expression of ribosomal protein genes in Saccharomyces cerevisiae but by different mechanisms.
Mol. Cell. Biol. 15: 3187-3196.

Oliver, E.R., Saunders, T.L., Tarle, S.A., and Glaser, T. 2004. Ribosomal protein L24 defect in Belly spot and tail (Bst), a mouse Minute. Development 131: 3907-3920.

Pestov, D.G., Strezoska, Z., and Lau, L.F. 2001. Evidence of p53-dependent cross-talk between ribosome biogenesis and the cell cycle: Effects of nucleolar protein Bop1 on G1/S transition. Mol. Cell. Biol. 21: 4246-4255.

Peyroche, G., Milkereit, P., Bischler, N., Tschochner, H., Schultz, P., Sentenac, A., Carles, C., and Riva, M. 2000. The recruitment of RNA polymerase I on rDNA is mediated by the interaction of the A43 subunit with Rrn3. EMBO I. 19: 5473-5482.

Polymenis, M. and Schmidt, E.V. 1997. Coupling of cell division to cell growth by translational control of the G1 cyclin CLN3 in yeast. Genes \& Dev. 11: 2522-2531.

Rubbi, C.P. and Milner, J. 2003. Disruption of the nucleolus mediates stabilization of p53 in response to DNA damage and other stresses. EMBO T. 22: 6068-6077.

Rupes, I. 2002. Checking cell size in yeast. Trends Genet. 18: $479-485$.

Saldanha, A.J., Brauer, M.J., and Botstein, D. 2004. Nutritional homeostasis in batch and steady-state culture of yeast. Mol. Biol. Cell 15: 4089-4104.

Saucedo, L.J. and Edgar, B.A. 2002. Why size matters: Altering cell size. Curr. Opin. Genet. Dev. 12: 565-571.

Stewart, M.J. and Denell, R. 1993. Mutations in the Drosophila gene encoding ribosomal protein S6 cause tissue overgrowth. Mol. Cell. Biol. 13: 2524-2535.

Sugimoto, M., Kuo, M.L., Roussel, M.F., and Sherr, C.J. 2003. Nucleolar Arf tumor suppressor inhibits ribosomal RNA processing. Mol. Cell 11: 415-424.

Thuriaux, P., Nurse, P., and Carter, B. 1978. Mutants altered in the control coordinating cell division with cell growth in the fission yeast Schizosaccharomyces pombe. Mol. Gen. Genet. 161: 215-220.

Toda, T., Cameron, S., Sass, P., and Wigler, M. 1988. SCH9, a gene of Saccharomyces cerevisiae that encodes a protein distinct from, but functionally and structurally related to, cAMP-dependent protein kinase catalytic subunits. Genes \& Dev. 2: 517-527.

Volarevic, S., Stewart, M.J., Ledermann, B., Zilberman, F., Terracciano, L., Montini, E., Grompe, M., Kozma, S.C., and Thomas, G. 2000. Proliferation, but not growth, blocked by conditional deletion of $40 \mathrm{~S}$ ribosomal protein S6. Science 288: 2045-2047.

Wade, C., Shea, K.A., Jensen, R.V., and McAlear, M.A. 2001. EBP2 is a member of the yeast $\mathrm{RRB}$ regulon, a transcriptionally coregulated set of genes that are required for ribosome and rRNA biosynthesis. Mol. Cell. Biol. 21: 8638-8650.

Warner, J.R. 1999. The economics of ribosome biosynthesis in yeast. Trends Biochem. Sci. 24: 437-440.

Watson, K.L., Konrad, K.D., Woods, D.F., and Bryant, P.J. 1992. Drosophila homolog of the human S6 ribosomal protein is required for tumor suppression in the hematopoietic system. Proc. Natl. Acad. Sci. 89: 11302-11306.

Zhang, Y., Yu, Z., Fu, X., and Liang, C. 2002. Noc3p, a bHLH protein, plays an integral role in the initiation of DNA replication in budding yeast. Cell 109: 849-860. 


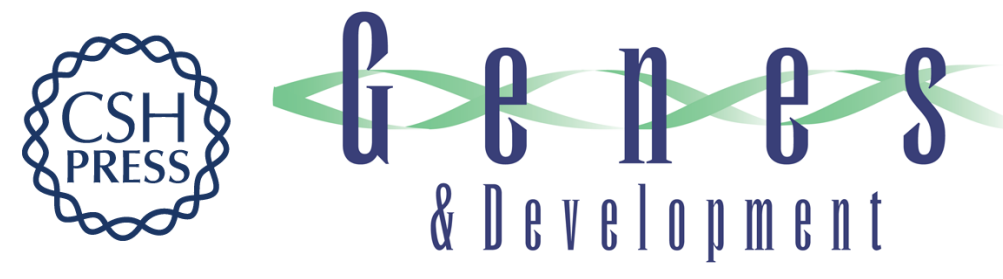

\section{What better measure than ribosome synthesis?}

Dipayan Rudra and Jonathan R. Warner

Genes Dev. 2004, 18:

Access the most recent version at doi:10.1101/gad.1256704

References This article cites 51 articles, 28 of which can be accessed free at: http://genesdev.cshlp.org/content/18/20/2431.full.html\#ref-list-1

License

Email Alerting Receive free email alerts when new articles cite this article - sign up in the box at the top Service right corner of the article or click here.

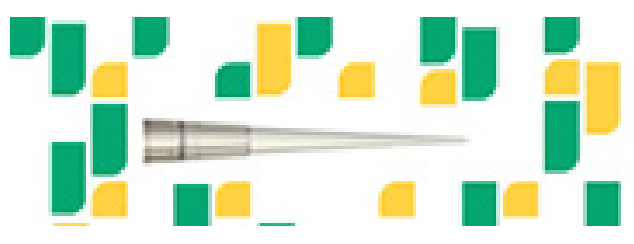

Focused on your science. 